\title{
Comparaison de plusieurs supports analytiques inertes et biologiques pour apprécier la contamination métallique de la retenue du site électronucléaire de Cattenom, France
}

\author{
J. Mersch 1 \\ N. Dubost 1 \\ J.C. Pihan 1
}

Mots clés : Métaux lourds, accumulation, indicateur, sédiment, mousse aquatique, moule zébrée, gardon, perche.

Les micropolluants métalliques $\mathrm{Cd}, \mathrm{Cu}, \mathrm{Pb}$ et $\mathrm{Zn}$ ont été analysés dans plusieurs compartiments inertes et biologiques de l'écosystème de la retenue du Mirgenbach : l'eau, les matières en suspension, le seston décanté, une mousse, un mollusque et trois organes de deux espèces dominantes de poissons. Ce lac artificiel reçoit les eaux échauffées de la centrale nucléaire de Cattenom (Nord-est de la France). L'étang de Lindre et la Meuse amont ont été choisis comme sites de référence.

L'ensemble des supports analytiques montre que la retenue est fortement contaminée par le cuivre et à un degré moindre par le plomb et le zinc. Des teneurs importantes de cadmium ont seulement été détectées dans le seston décanté. Les métaux entrant dans la retenue se partagent entre deux phases : la colonne d'eau et les sédiments. Les concentrations élevées observées dans le seston décanté indiquent qu'une fraction importante de chaque métal disparaît de la phase circulante et stagne dans la retenue. Dans le cas du cuivre, une estimation des flux révèle que les deux tiers de ce métal se retrouvent dans les matières décantées. L'homogénéité dans la détection des différents micropolluants dans la moule Dreissena polymorpha Pallas et la mousse Fontinalis antipyretica Hedw. confirme l'intérêt de ces deux organismes comme indicateurs biologiques de la pollution circulante. Les deux poissons, le gardon Rutilus rutilus L. et la perche Perca fluviatilis L., se distinguent par de faibles concentrations métalliques dans le muscle. Bien que certains métaux aient été accumulés dans les organes cibles comme le foie pour le cuivre et le rein pour le plomb, ils peuvent difficilement être considérés comme des organismes sentinelles.

Comparison of several inert and biological substrata to assess the trace metal contamination in the reservoir of the nuclear power plant in Cattenom, France

Keywords : Heavy metals, accumulation, indicator, sediment, aquatic bryophyte, zebra mussel, roach, perch.

The trace metals $\mathrm{Cd}, \mathrm{Cu}, \mathrm{Pb}$ and $\mathrm{Zn}$ were analyzed in the following inert and biological compartments of the ecosystem of the Mirgenbach reservoir : the water, the suspended solids, the settling particulate matter, a bryophyte, a mollusc and three organs of two dominant fish species. This man-made lake receives the warmed waters from the nuclear power plant in Cattenom (Northeastern France). The Lindre pond and an upper section of the Meuse River were chosen as reference sites.

All the analyzed substrata showed that the Mirgenbach reservoir is strongly contaminated with copper and moderately with lead and zinc. High burdens of cadmium were only detected in the sediment samples. The trace metals entering the reservoir partition between two different phases : the-water column and the sediments. The elevated concentrations observed in the settling particulate matter indicate that a large amount of each metal is eliminated from the water. In the particular case of copper, estimations of fluxes showed that two thirds of the discharged metal were trapped in the sediments. The good agreement of metal detection in the mussel Dreissena polymorpha Pallas and in the moss Fontinalis antipyretica Hedw. emphasizes the suitability of both organisms to be used as biological indicators for water pollution. The two fish species, the roach Rutilus rutilus $\mathrm{L}$. and the perch Perca fluviatilis L., exhibited low metal concentrations in their muscle flesh. Although accumulation occurred in the target tissues, e.g. copper in the liver and lead in the kidney, fish cannot be considered as adequate sentinel organisms.

1. Centre de Recherches Ecologiques de l'Université de Metz, Laboratoire d'Ecotoxicologie, B.P. 4116, 57040 Metz Cedex 01, France.

Texte d'une communication effectuée dans le cadre du Colloque - Limnologie appliquée et application de la Limnologie - Besançon, Franche-Comté, 16-19 novembre 1992. 


\section{Introduction}

La recherche et la quantification de micropolluants peuvent être réalisées dans différents compartiments d'un écosystème. Deux types de supports analytiques sont à distinguer : les supports inertes et les supports biologiques. Les supports inertes comme l'eau (brute ou filtrée), les matières en suspension ou les sédiments renseignent sur la charge totale en contaminants d'un milieu. L'eau et les matières en suspension ont cependant l'inconvénient de ne fournir qu'une image instantanée de l'état de contamination d'un site. Le sédiment, par contre, conserve l'empreinte d'une pollution jusqu'à une éventuelle remobilisation (Nakanishi et al. 1989, Tanner \& Clayton 1990). Ses capacités d'indicateur de pollution se heurtent tout de même au problème de dynamique de dépôt qui rend difficile un prélèvement représentatif d'une période donnée. Le second type de supports analytiques, les organismes vivants, présente l'avantage d'estimer la fraction biodisponible des contaminants. De plus, la réponse obtenue est intégrée dans le temps et rend compte de la contamination moyenne récente d'un site. Parmi les organismes proposés comme indicateurs biologiques de pollution chimique en milieu dulçaquicole, les bryophytes aquatiques et les mollusques filtreurs ont particulièrement retenu l'attention en Europe (Wehr et al. 1983, Kelly et al. 1987, Léglize $\&$ Crochard 1987, Mersch et al. 1992). Pour des raisons de santé publique, mais aussi en tant qu'organismes plus complexes, les poissons font également l'objet d'analyses de micropolluants (VillarrealTreviño et al. 1986, Pfeiffer et al. 1989, Guerrin et al. 1990, Gonzáles et al. 1991).

Dans la plupart des travaux in situ, notamment dans le cadre de la surveillance du milieu naturel, un seul support analytique est utilisé. L'indicateur retenu est alors jugé représentatif de l'ensemble du système étudié. Or, le choix de l'indicateur reste empirique et des études comparées s'imposent afin de valider et de juger de l'intérêt de différents supports analytiques. Dans le présent travail, les analyses de micropolluants métalliques $\mathrm{Cd}, \mathrm{Cu}, \mathrm{Pb}$ et $\mathrm{Zn}$ ont porté sur : l'eau brute, les matières en suspension, le seston décanté, la mousse aquatique Fontinalis antipyretica Hedw., la moule zébrée Dreissena polymorpha Pallas ainsi que le muscle, le foie et le rein de deux espèces de poisson : un planctonophage, le gardon Rutilus rutilus L. et un carnassier, la perche Perca fluviatilis L.. L'objectif consiste donc à suivre la répartition de contaminants métalliques dans divers compartiments d'un écosystème et à évaluer la signification écotoxicologique ainsi que l'intérêt comparé des différents supports analytiques utilisés.

\section{Matériel et méthodes}

\subsection{Description des sites d'étude}

La retenue du Mirgenbach est un réservoir artificiel d'une centaine d'hectares et d'une capacité de $7.310^{6} \mathrm{~m}^{3}$. Elle sert de tampon thermique et de réserve d'eau de sauvegarde aux 4 réacteurs d'une puissance unitaire de $1300 \mathrm{MW}$ de la centrale nucléaire de Cattenom (Moselle, France). L'eau de refroidissement $\left(8.8 \mathrm{~m}^{3} . \mathrm{s}^{-1}\right)$ est directement prélevée dans la rivière Moselle. Les aéroréfrigérants évaporent $3.0 \mathrm{~m}^{3} . \mathrm{s}^{-1}$ et les eaux de purge $\left(5.0 \mathrm{~m}^{3} . \mathrm{s}^{-1}\right)$ $\mathrm{du}$ circuit de refroidissement tertiaire, enrichies en sels minéraux, sont injectées, avec des débits annexes, dans la retenue au niveau d'un ouvrage de rejet. Le temps de séjour moyen de l'eau est d'environ 10 jours pour le fonctionnement des 4 réacteurs. Un déversoir permet un débit sortant équivalent vers la Moselle. En réalité, la gestion de ce réservoir est plus complexe et plusieurs options s'offrent à l'exploitant qui peut ou non fonctionner en boucle sur la retenue ou bien court-circuiter celle-ci. L'eau prélevée en rivière est soumise à un traitement mécanique de dégrillage puis de filtration sur tambour. Dans ces conditions, le flux de pollution métallique entrant dans la retenue ne peut être valablement calculée à partir des concentrations à la prise d'eau en rivière.

Les micropolluants métalliques de la retenue ont deux origines possibles : les eaux de la Moselle qui drainent une grande partie du bassin sidérurgique lorrain et les circuits tertiaires de refroidissement de la centrale. Des analyses de métaux réalisées en janvier 1989 dans un organisme indicateur autochtone, la moule zébrée Dreissena polymorpha, ont révélé une contamination du secteur de la Moselle situé en amont de Cattenom par le plomb et dans une moindre mesure par le zinc. En revanche, les concentrations de cadmium et de cuivre sont restées faibles (Mersch et al. 1992). Lors de ce même travail, les moules ont également été prélevées dans la retenue du Mirgenbach. Elles y ont indiqué une contamination par le cuivre, mais aussi par le cadmium et le zinc. Le cuivre provient de phénomènes de corrosion et d'abrasion des tubes en laiton (alliage de 
Cu et de $\mathrm{Zn}$ ) des échangeurs du circuit tertiaire de refroidissement. Compte tenu de sa présence dans la Moselle et comme composante du laiton, le zinc a certainement une origine mixte. Enfin, le cadmium pourrait provenir de la corrosion de surfaces métalliques cadmiées actuellement non identifiées.

Afin d'évaluer le degré de pollution des différents compartiments de la retenue du Mirgenbach, des analyses ont été réalisées sur les mêmes supports de sites non contaminés. Le premier site de référence choisi est le Domaine Piscicole de Lindre (Moselle). Il s'agit également d'un plan d'eau artificiel. Comme la pisciculture extensive nécessite des vidanges régulières de l'étang, aucune population de moules zébrées ne peut s'y établir. La station de référence pour ce mollusque est un secteur canalisé de l'amont de la rivière Meuse. Les mousses aquatiques qui se développent préférentiellement en eau courante ont été récoltées sur une surverse de ce même canal.

\subsection{Prélèvements}

L'effet de concentration dans la retenue du Mirgenbach par rapport à la Moselle a été évalué par un suivi mensuel, entre mars 1991 et février 1992, de la conductivité de l'eau et des teneurs en deux ions majeurs, $\mathrm{Na}^{+}$et $\mathrm{Cl}-$. L'étude du niveau de contamination du réservoir est basée sur des prélèvements mensuels d'eau brute et de dreissènes autochtones pendant la même période. Les moules ont été récoltées sur un support immergé en pleine eau. Lors de neuf campagnes, des échantillons supplémentaires d'eau de surface ont été collectés afin de déterminer la répartition des métaux entre la phase solide (matières en suspension) et la phase libre. A trois reprises entre juillet et décembre 1991, des sédimentomètres composés d'un bidon de $50 \mathrm{l}$ surmonté d'un cône collecteur ont été placés sur le fond de plusieurs stations de la retenue. La matière décantée recueillie a été récoltée après 15 jours. Une touffe de mousse d'environ $20 \mathrm{~g}$ (poids essoré) a été transférée de la Meuse dans la retenue du Mirgenbach. Elle a été immergée à l'intérieur d'un sachet grillagé fixé à une bouée adjacente au support des mollusques. Le temps d'exposition a été de deux semaines entre le 13 et le 27 novembre. La technique de transfert de bryophytes aquatiques a été décrite par Kelly et al. (1987). Enfin, les poissons ont été pêchés à l'aide de filets verticaux fin octobre 1991. Cinq individus des deux espèces dominantes de la retenue, le gardon et la perche, ont été utilisés pour l'analyse des micropolluants métalliques. Au moment des prélèvements de juillet à décembre 1991, les quatre réacteurs de la centrale étaient en service.

L'effort d'échantillonnage sur les deux sites de référence a été moindre car, d'une part, les niveaux de contamination métallique dans les différents compartiments de ces milieux peuvent être considérés comme plus stables, en raison de leur localisation dans une région à dominance agricole ; d'autre part, des données bibliographiques concernant des sites semblables peuvent être utilisées en complément. Le matériel de comparaison originaire du Domaine Piscicole de Lindre est constitué de trois échantillons d'eau brute, de deux échantillons de matières décantées ainsi que de quatre poissons des deux espèces. Un seul échantillon de mousses (correspondant au témoin avant transfert) et de moule a été récolté dans la Meuse.

\subsection{Préparation des échantillons et analyse}

La conductivité de l'eau de la Moselle et de la retenue du Mirgenbach est mesurée sur place à l'aide d'une sonde VTW. L'analyse des ions $\mathrm{Na}+$ et $\mathrm{Cl}-$ est réalisée sur de l'eau brute. Les chlorures sont dosés par chromatographie ionique après dilution. Les concentrations de sodium sont déterminées par spectrophotométrie d'émission atomique. L'eau brute destinée à l'analyse des micropolluants métalliques est prélevée dans des flacons de $500 \mathrm{ml}$ en polyéthylène et acidifiée à $1 \%(\mathrm{v} / \mathrm{v})$ avec de l'acide nitrique (normapur). Les échantillons sont stockés en chambre froide à $4^{\circ} \mathrm{C}$ jusqu'au dosage. Afin de déterminer la fraction métallique libre et celle associée aux matières en suspension, $300 \mathrm{ml}$ d'eau brute prélevés en surface dans une bouteille de verre sont filtrés sur une membrane en nitrate de cellulose d'un diamètre de pore de $0.45 \mu \mathrm{m}$, préalablement rincé dans un bain acide. Le filtrat est traité comme l'eau brute et le filtre est minéralisé selon le même protocole que les échantillons biologiques.

La matière décantée recueillie dans les sédimentomètres est séchée à l'étuve à $90^{\circ} \mathrm{C}$ pendant 24 heures et broyée au mortier jusqu'à obtention d'une poudre homogène. Aucun tamisage ne s'est avéré nécessaire. L'extraction des métaux dans $1 \mathrm{~g}$ de matière sèche est réalisée en deux étapes, successivement avec $30 \mathrm{ml}$ d'acide nitrique à $36 \%$ et un mélange de $10 \mathrm{ml}$ d'acide nitrique à $36 \%$ et $10 \mathrm{ml}$ d'acide perchlorique à $35 \%$ (qualités normapur). Le temps 
d'extraction à froid sur table d'agitation est de 24 heures. Les deux surnageants sont réunis et réduits sur plaque chauffante à $250^{\circ} \mathrm{C}$ à un volume de 1 à $2 \mathrm{ml}$. Le volume est ensuite ajusté à $50 \mathrm{ml}$ avec de l'eau distillée acidifiée à $4 \%$ par de l'acide nitrique. Le procédé est répété sur deux souséchantillons indépendants. Ce protocole utilisant des acides forts permet une extraction complète des métaux associés aux sédiments (Batley 1987, Tessier \& Campbell 1987).

Les échantillons biologiques, c'est-à-dire les bryophytes et les parties molles des moules zébrées in toto ainsi que le muscle, le foie et le rein des gardons et des perches, sont soumis à un même protocole de minéralisation. Les organes de poissons sont analysés individuellement alors que chaque touffe de mousse est divisée en deux sous-échantillons et que les moules sont séparées en deux lots de douze spécimens. La préparation des bryophytes comporte une étape de rinçage sous un jet d'eau déminéralisée (Wehr et al. 1983). Les mollusques et les poissons sont disséqués. Les échantillons sont ensuite séchés à $40^{\circ} \mathrm{C}$ et minéralisés $(24 \mathrm{~h})$ à chaud $\left(70^{\circ} \mathrm{C}\right)$ et sous pression dans des tubes en polyéthylène en utilisant $2 \mathrm{ml}$ d'acide nitrique à $36 \%$ (normapur) pour une masse de $200 \mathrm{mg}$ de tissu. Le volume est ajusté à $19 \mathrm{ml}$ avec de l'eau distillée pour obtenir une solution à $4 \%$ d'acide. Dans certains cas, la masse sèche disponible est inférieure à $200 \mathrm{mg}$. Les volumes utilisés sont alors calculés en conséquence afin d'obtenir la même matrice à soumettre au dosage. Des essais à blanc, inclus dans chaque série de dosage et traités de la même façon que les minéralisats, sont destinés à évaluer l'apport métallique du matériel et des réactifs. L'analyse des métaux $\mathrm{Cd}, \mathrm{Cu}, \mathrm{Pb}$ et $\mathrm{Zn}$ est effectuée par spectrophotométrie d'absorption atomique en flamme ou en four selon les concentrations. Des ajouts dosés sont effectués pour détecter d'éventuels effets de matrice.

\subsection{Statistiques}

Le traitement statistique des données utilisé est le test $t$ de Student de comparaison de moyennes. Le seuil de significativité est fixé à $5 \%$.

\section{Résultats}

\subsection{Eau}

Le suivi mensuel, entre mars 1991 et février 1992, de la conductivité de l'eau et des ions majeurs, $\mathrm{Na}^{+}$ et $\mathrm{Cl}^{-}$, met en évidence l'effet de concentration dans la retenue par rapport à la Moselle, suite à l'évaporation d'une partie de l'eau dans les aéroréfrigérants (Tableau 1). Les facteurs de concentration moyens sont respectivement $1.4,1.5$ et 1.3 pour la conductivité, les chlorures et le sodium. La minéralisation de la Moselle est élevée $\left(1650 \mu \mathrm{S} . \mathrm{cm}^{-1}\right.$ en moyenne) avec des fluctuations d'une amplitude d'environ $400{ }_{\mu} \mathrm{S} . \mathrm{cm}^{-1}$. Ces fluctuations sont amplifiées au niveau de la retenue avec un maximum de $930{ }_{\mu} \mathrm{S} . \mathrm{cm}^{-1}$. Les écarts de minéralisation entre la rivière et le réservoir sont directement liés au mode d'exploitation des ressources en eau par la centrale.

Tableau 1. Suivi mensuel de la conductivité et des concentrations en $\mathrm{Na}^{+}$et $\mathrm{Cl}^{-}$de l'eau de la rivière Moselle et la retenue du Mirgenbach.

Table 1. Monthly measurements of conductivity and concentrations of $\mathrm{Na}^{+}$and $\mathrm{Cl}^{-}$in the water of the Moselle River and the Mirgenbach reservoir.

\begin{tabular}{|c|c|c|c|c|}
\hline Station & $\begin{array}{l}\text { prélèvement } \\
\text { (mois) }\end{array}$ & $\begin{array}{l}\text { conductivité } \\
(\mu \mathrm{S} / \mathrm{cm})\end{array}$ & $\begin{array}{r}\mathrm{Na}^{+} \\
(\mathrm{mg} / \mathrm{L})\end{array}$ & $\begin{array}{c}\mathrm{Cl}^{-} \\
(\mathrm{mg} / \mathrm{L})\end{array}$ \\
\hline Rivière & 03.91 & 1420 & 120 & 380 \\
\hline \multirow[t]{8}{*}{ Moselle } & 04.91 & 1475 & 130 & 390 \\
\hline & 05.91 & 1780 & 160 & 500 \\
\hline & 06.91 & 1650 & 160 & 440 \\
\hline & 07.91 & 1710 & 160 & 485 \\
\hline & 08.91 & 1800 & 180 & 525 \\
\hline & 09.91 & 1635 & 165 & 440 \\
\hline & 10.91 & 1540 & 150 & 440 \\
\hline & 11.91 & 1700 & 135 & 505 \\
\hline \multirow[t]{3}{*}{$\therefore$} & 12.91 & 1820 & 170 & 525 \\
\hline & 01.92 & 1710 & 145 & 460 \\
\hline & 02.92 & 1550 & 115 & 415 \\
\hline \multirow{2}{*}{$:$} & moyenne & 1650 & 150 & 460 \\
\hline & \pm écart-type & 130 & 20 & 50 \\
\hline Retenue du & 03.91 & 1800 & 120 & 500 \\
\hline \multirow[t]{13}{*}{ Mirgenbach } & 04.91 & 1810 & 140 & 495 \\
\hline & -05.91 & 1800 & 130 & 495 \\
\hline & 06.91 & 1930 & 150 & 555 \\
\hline & 07.91 & 2330 & 215 & 705 \\
\hline & 08.91 & 2540 & 255 & 800 \\
\hline & 09.91 & 2730 & 275 & 820 \\
\hline & 10.91 & 2710 & 265 & 805 \\
\hline & 11.91 & 2150 & 160 & 605 \\
\hline & 12.91 & 2310 & 180 & 680 \\
\hline & 01.92 & 2710 & 235 & 810 \\
\hline & 02.92 & 2690 & 185 & 820 \\
\hline & moyenne & 2295 & 195 & 675 \\
\hline & \pm écart-type & 385 & 55 & 140 \\
\hline
\end{tabular}


Pour trois des quatre métaux analysés $(\mathrm{Cu}, \mathrm{Pb}$ et $\mathrm{Zn}$ ), les concentrations sont significativement plus élevées (test $t$ ) dans les échantillons d'eau de la retenue du Mirgenbach que dans ceux du site témoin, représenté par l'étang de Lindre (Fig. 1). L'écart le plus important est observé pour le cuivre.

Ces résultats peuvent être confrontés à des données plus générales, comme les concentrations de référence établies par l'Agence de l'Eau Rhin-Meuse (1991) pour les eaux régionales : $\mathrm{Cd}: 0.2, \mathrm{Cu}: 3$,

Cd

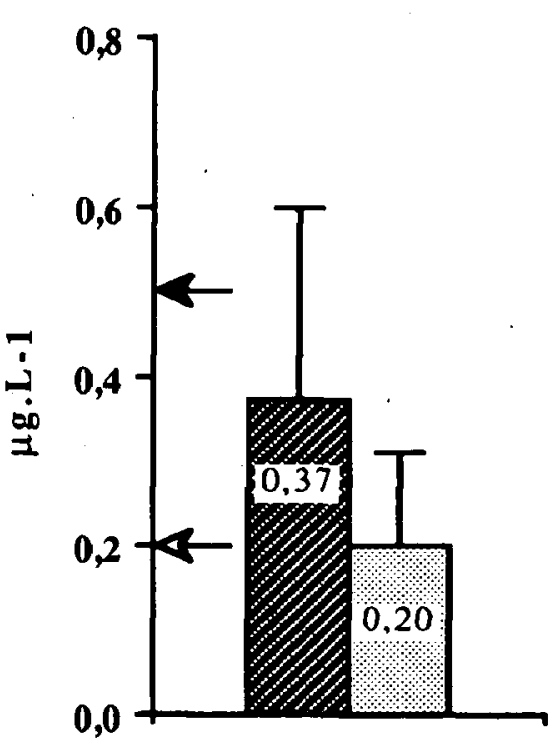

$\mathrm{Cu}$

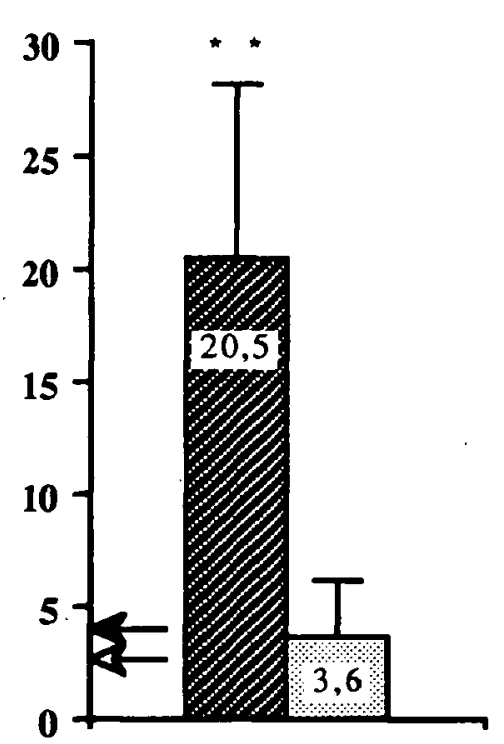

$\mathrm{Pb}: 5$ et $\mathrm{Zn}: 30 \mu \mathrm{g} .1^{-1}$. Par rapport à ces valeurs, la contamination de la retenue par le cuivre est confirmée. Il en est de même, si l'on compare les résultats aux concentrations géochimiques considérées comme niveaux métalliques moyens dans les eaux européennes : $\mathrm{Cd}: 0.5, \mathrm{Cu}: 4, \mathrm{~Pb}: 2.5$ et $\mathrm{Zn}$ : $20 \mu \mathrm{g} .1^{-1}$ (Förstner \& Wittmann 1981). Les écarts entre les données régionales et européennes, notamment pour le plomb et le zinc, s'expliquent par l'activité sidérurgique de la Lorraine.
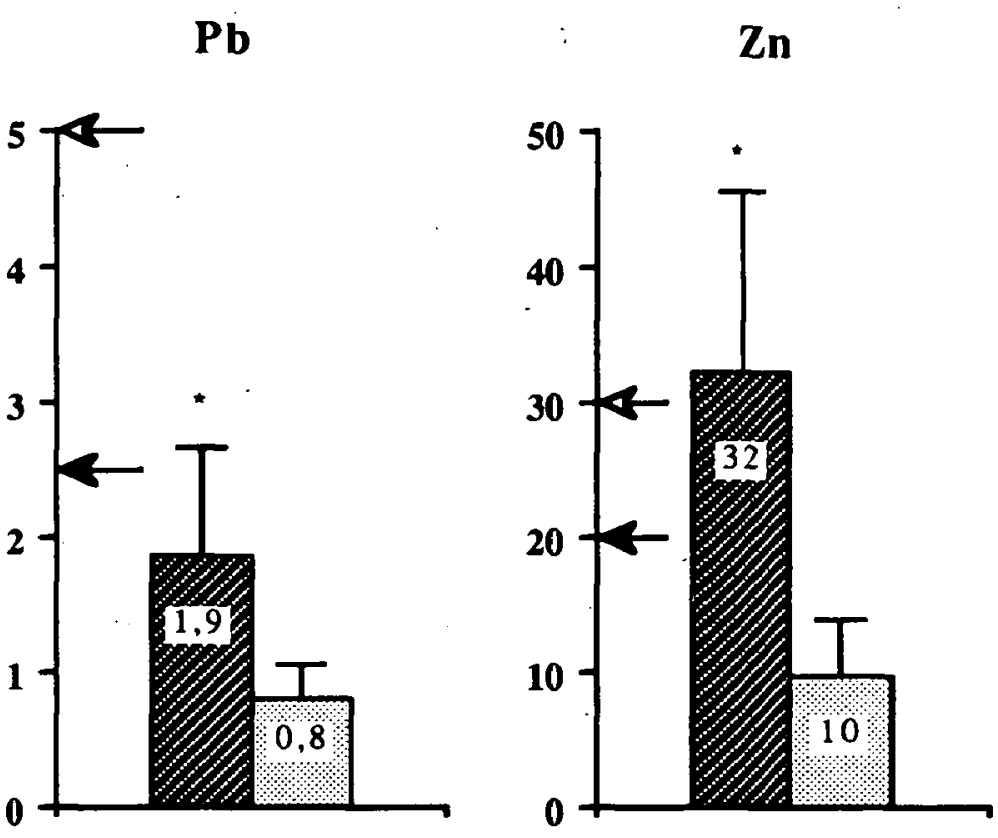

Fig. 1. Concentrations en $\mathrm{Cd}, \mathrm{Cu}, \mathrm{Pb}$ et $\mathrm{Zn}$ dans l'eau $\left(\mu \mathrm{g} .1^{-1}\right)$ de la retenue du Mirgenbach (barres foncées ; $n=6$ ) et de l'étang de Lindre (barres claires ; $n=3$ ). Données de référence : Förstner \& Wittmann 1981 (flèche noire). Agence de l'Eau Rhin-Meuse 1991 (flèche blanche) ; ${ }^{*}: p<0.05,^{* *}: p<0.01$.

Fig. 1. Concentrations of $\mathrm{Cd}, \mathrm{Cu}, \mathrm{Pb}$ and $\mathrm{Zn}$ in the water $\left(\mu \mathrm{g} .1^{-1}\right)$ of the Mirgenbach reservoir (dark bars ; $\left.n=6\right)$ and of the Lindre pond (light bars ; $n=3$ ). Reference data : Förstner \& Wittmann 1981 (black arrow), Agence de l'Eau Rhin-Meuse 1991 (white arrow) $;^{*}: p<0.05,{ }^{* *}: p=<0.01$.

\subsection{Matières en suspension}

Dans des échantillons d'eau brute collectés en surface de la retenue du Mirgenbach, les fractions de cuivre et de zinc associées aux matières en suspension représentent respectivement $26 \pm 7 \%(n=9)$ et $28 \pm 2 \%(n=3)$ du métal total. Environ $75 \%$ de ces deux métaux se trouvent donc sous forme libre dans l'eau ou alors adsorbés à des particules de taille inférieure à $0.45 \mu \mathrm{m}$. En ce qui concerne le plomb et le cadmium, leurs faibles concentrations rendent difficile une expression en pourcentages ; le plomb est fortement lié à la phase solide alors que le cadmium reste très majoritairement libre.

\subsection{Seston décanté}

Les différences entre les concentrations en métaux dans la retenue du Mirgenbach et l'étang de Lindre sont significatives au seuil de $1 \%$ pour tous les métaux (Fig. 2). Les niveaux métalliques mesurés dans les matières décantées de l'étang de Lindre sont comparables avec les valeurs de référence régionales, respectivement $0.5,20,20$ et $75 \mu \mathrm{g} . \mathrm{g}^{-1}$ de poids sec pour les métaux $\mathrm{Cd}, \mathrm{Cu}, \mathrm{Pb}$ et $\mathrm{Zn}$ (Agence de l'Eau Rhin-Meuse 1991). Elles le sont également avec les teneurs métalliques médianes dans les sédiments collectés dans 87 lacs répartis sur tous les continents : $\mathrm{Cu}: 45, \mathrm{~Pb}: 34$ et $\mathrm{Zn}: 118 \mu \mathrm{g}-\mathrm{g}-1$ (Förstner \& Wittmann 1981). Ces données mettent en évidence l'importance du niveau de contamination du seston décanté de la retenue du Mirgenbach.

Les écarts-types inter-échantillons très faibles (Fig. 2) indiquent que les concentrations en métaux dans les matières décantées ont été homogènes au cours du temps, quel que soit le lieu de récolte dans la retenue. 

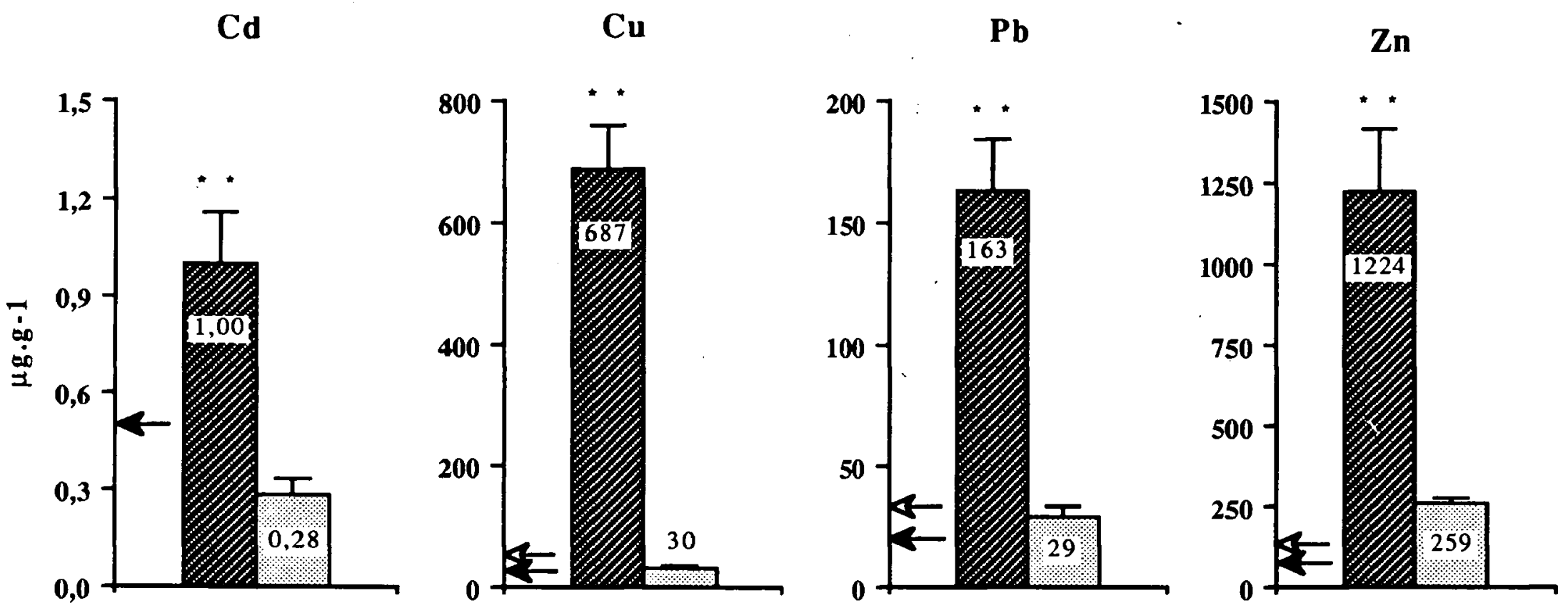

Fig. 2. Concentrations en $\mathrm{Cd}, \mathrm{Cu}, \mathrm{Pb}$ et $\mathrm{Zn}$ dans le seston décanté ( $\mu \mathrm{g} \cdot \mathrm{g}^{-1}$ de poids sec) de la retenue du Mirgenbach (barres foncées ; $n=9$ ) et de l'étang de Lindre (barres claires ; $n=2$ ). Données de référence : Agence de l'Eau Rhin-Meuse 1991 (flèche noire), Förstner \& Wittmann 1981 (flèche blanche) ; ${ }^{* *}: p<0.01$.

Fig. 2. Concentrations of $\mathrm{Cd}, \mathrm{Cu}, \mathrm{Pb}$ and $\mathrm{Zn}$ in the settling particulate matter ( $\mu \mathrm{g} \cdot \mathrm{g}^{-1}$ dry weight) of the Mirgenbach reservoir (dark bars ; $n=9$ ) and of the Lindre pond (light bars ; $n=2$ ). Reference data : Agence de l'Eau Rhin-Meuse 1991 (black arrow), Förstner \& Wittmann 1981 (white arrow); ${ }^{* *}: p<0.01$.

En revanche, la répartition spatiale des dépôts est plus irrégulière. Les quantités, exprimées en poids sec, ont en effet varié de 16 à $80 \mathrm{~g} . \mathrm{j}^{-1} \cdot \mathrm{m}^{-2}$ avec une moyenne de $48 \pm 23 \mathrm{~g} . \mathrm{j}^{-1} \cdot \mathrm{m}^{-2}(n=9)$. Contrairement aux sédiments de fond, qui peuvent subir des transports verticaux et horizontaux, la matière recueillie dans les sédimentomètres est représentative d'une période et d'une station données. Il est par conséquent possible de calculer des flux de métaux déposés. Ceux-ci sont obtenus en multipliant pour chaque échantillon la concentration moyenne (Fig. 2) par la masse moyenne de seston récolté, soit : $\mathrm{Cd}: 48 \pm 23 \mu \mathrm{g} \cdot \mathrm{j}^{-1} \cdot \mathrm{m}^{-2}, \mathrm{Cu}: 33 \pm$ $17 \mathrm{mg} . \mathrm{j}^{-1} \cdot \mathrm{m}^{-2}, \mathrm{~Pb}: 7.9 \pm 3.9 \mathrm{mg} . \mathrm{j}^{-1} \cdot \mathrm{m}^{-2}$ et $\mathrm{Zn}: 59 \pm 27 \mathrm{mg} \cdot \mathrm{j}^{-1} \cdot \mathrm{m}^{-2}(n=8)$. En admettant que la sédimentation soit uniforme sur les 96 ha de la retenue, les dépôts s'élèveraient à $1.4 \pm 0.7 \mathrm{~kg}$ de Cd, $938 \pm 474 \mathrm{~kg}$ de $\mathrm{Cu}, 224 \pm 111 \mathrm{~kg}$ de $\mathrm{Pb}$ et $1684 \pm 780 \mathrm{~kg}$ de $\mathrm{Zn}$ par mois pour la période d'étude comprise entre juillet et décembre 1991.

\subsection{Mousse aquatique}

Après deux semaines d'immersion dans la retenue du Mirgenbach, les mousses Fontinalis antipyretica ont multiplié leur teneur en cuivre par 6.6 (Fig. 3). Elles indiquent en outre une contamination par le plomb et par le zinc. Par contre, il n'y a pas d'évo- lution pour le cadmium. Les charges métalliques dans les mousses de la station témoin de la Meuse sont en accord avec les niveaux de référence,suédois qui s'appliquent précisément au genre Fontinalis (Swedish Environmental Protection Agency 1991) : $\mathrm{Cd}: 0.5, \mathrm{Cu}: 10, \mathrm{~Pb}: 3$ et $\mathrm{Zn}: 100 \mu \mathrm{g} \cdot \mathrm{g}^{-1}$ de poids sec. Les références standard proposées par Mouvet (1986), respectivement 1, 19, 19 et 200 $\mu \mathrm{g} . \mathrm{g}^{-1}$, sont plus élevées. Ces références confirment la pollution de la retenue par le cuivre, mais suggèrent que les contaminations par le plomb et le zinc sont relativement faibles.

\subsection{Moule zébrée}

Les moules autochtones Dreissena polymorpha mettent également en évidence la contamination de la retenue du Mirgenbach par le cuivre (Fig. 4). Par rapport à la station de référence de la Meuse, la concentration moyenne est 6.8 fois plus élevée. De plus, les moules indiquent une contamination par le plomb et, à un moindre degré, par le zinc. Les concentrations métalliques dans des moules zébrées prélevées dans des lacs néerlandais, 1, 12, 0.5 et 110 $\mu \mathrm{g} . \mathrm{g}^{-1}$ de poids sec respectivement pour $\mathrm{Cd}, \mathrm{Cu}$, $\mathrm{Pb}$ et $\mathrm{Zn}$ (Kraak et al: 1991), sont très proches de celles des individus de la Meuse. Une deuxième série de valeurs géochimiques $\left(1,18,1.5\right.$ et $101 \mu \mathrm{g} \cdot \mathrm{g}^{-1}$; 
Cd

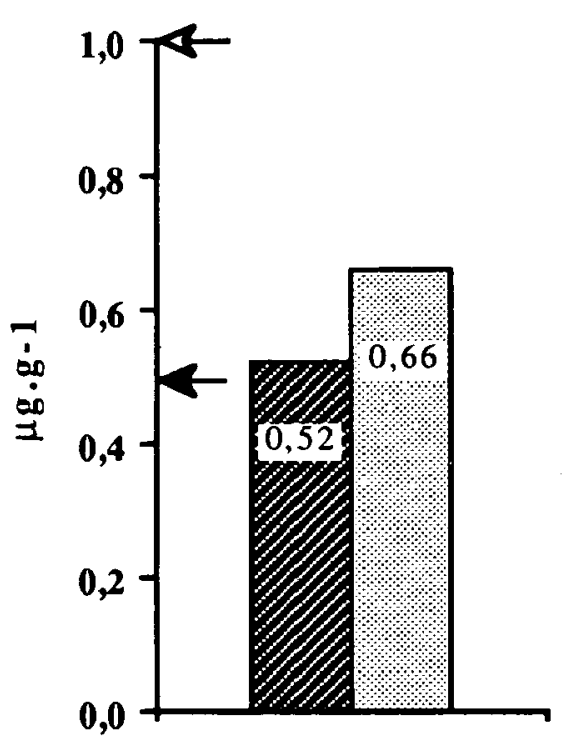

$\mathrm{Cu}$

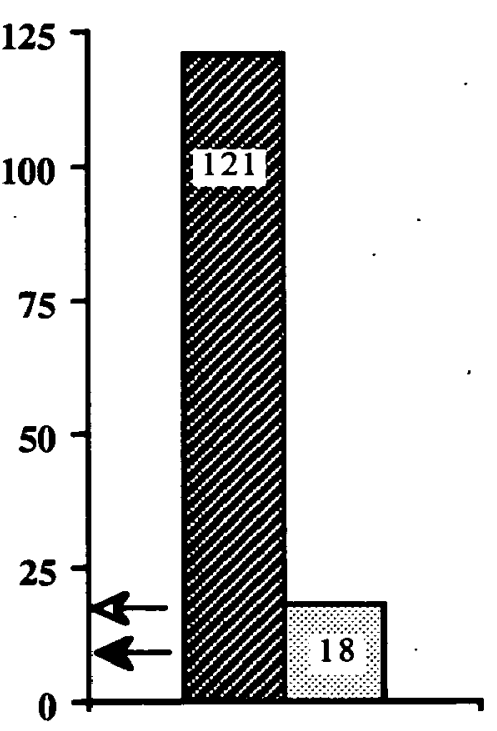

$\mathbf{P b}$

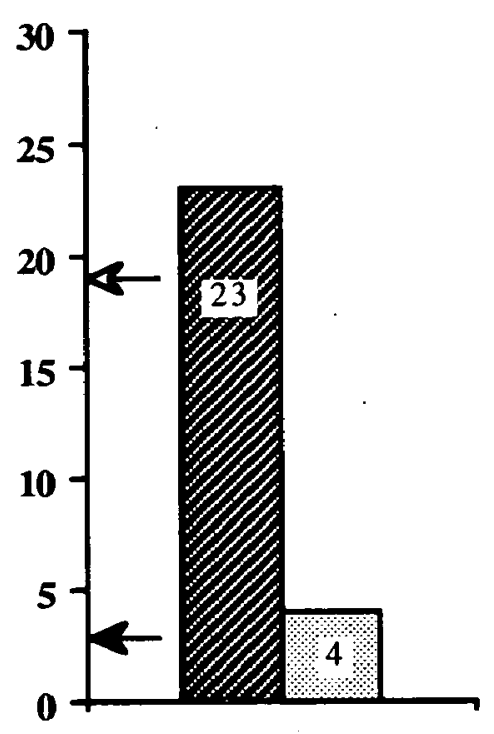

Zn

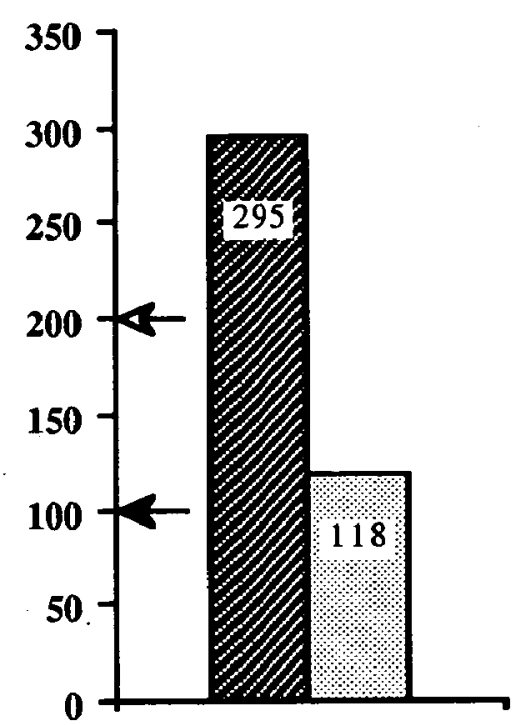

Fig. 3. Concentrations en $\mathrm{Cd}, \mathrm{Cu}, \mathrm{Pb}$ et $\mathrm{Zn}$ dans la mousse aquatique Fontinalis antipyretica ( $\mu \mathrm{g} . \mathrm{g}^{-1}$ de poids sec) transférée à partir de la Meuse (barres claires) dans la retenue du Mirgenbach (barres foncées). Données de référence : Swedish Environmental Protection Agency 1991 (flèche noire), Mouvet 1986 (flèche blanche).

Fig. 3. Concentrations of $\mathrm{Cd}, \mathrm{Cu}, \mathrm{Pb}$ and $\mathrm{Zn}$ in the aquatic moss Fontinalis antipyretica $\left(\mu \mathrm{g} . \mathrm{g}^{-1}\right.$ dry weight) transferred from the Meuse River (light bars) into the Mirgenbach reservoir (dark bars). Reference data : Swedish Environmental Protection Agency 1991 (black arrow), Mouvet 1986 (white arrow).

Cd

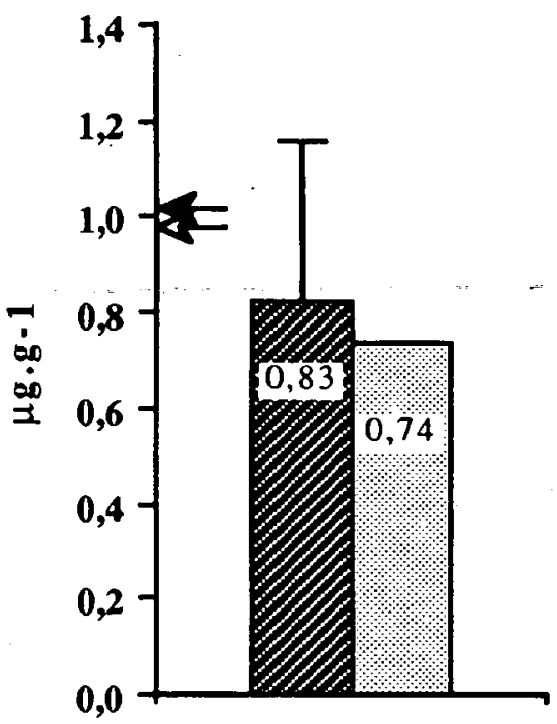

$\mathbf{C u}$

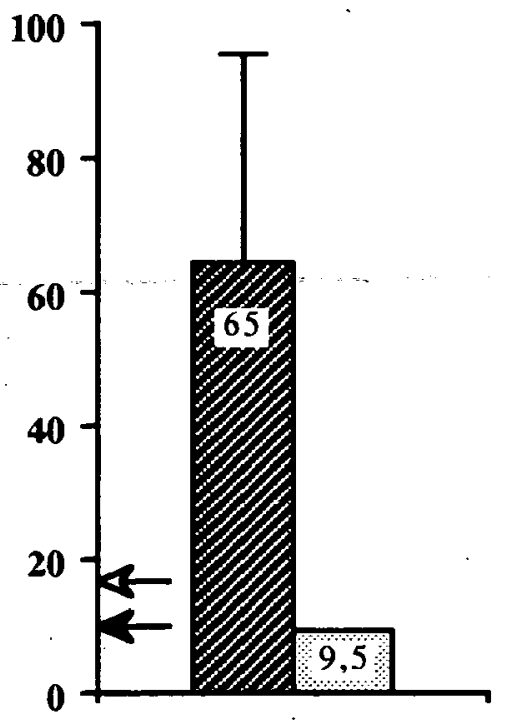

$\mathbf{P b}$

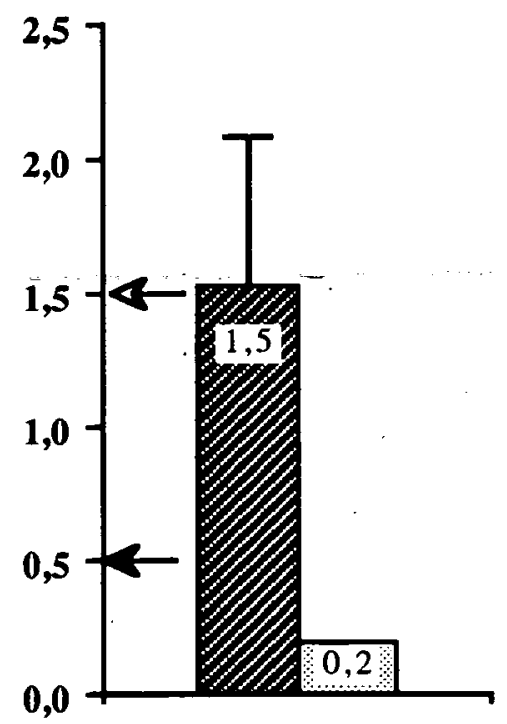

Zn

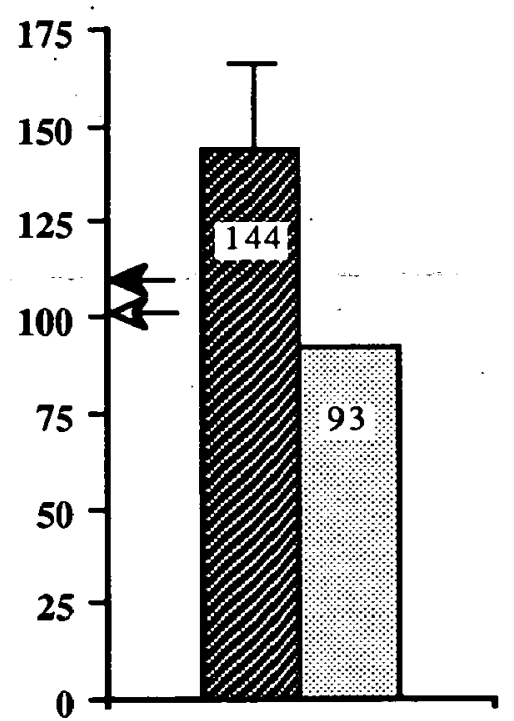

Fig. 4. Concentrations en $\mathrm{Cd}, \mathrm{Cu}, \mathrm{Pb}$ et $\mathrm{Zn}$ dans la mousse zébrée Dreissena polymorpha ( $\mu \mathrm{g} . \mathrm{g}^{-1} \mathrm{de}$ poids sec) de la retenue du Mirgenbach (barres foncées; $n=6$ ) et de la Meuse (barres claires; $n=1$ ). Données de référence : Kraak et al. 1991 (flèche noire), Mersch et al. 1992 (flèche blanche).

Fig. 4. Concentrations of $\mathrm{Cd}, \mathrm{Cu}, \mathrm{Pb}$ and $\mathrm{Zn}$ in the zebra mussel Dreissena polymorpha ( $\mu \mathrm{g} \cdot \mathrm{g}^{-1}$ dry weight) from the Mirgenbach reservoir (dark bars ; $n=6$ ) and from the Meuse River (light bars ; $n=1$ ). Reference data : Kraak et al. 1991 (black arrow), Mersch et al. 1992 (white arrow).

Mersch et al. 1992) est également applicable, sauf peut-être pour le plomb. La teneur élevée de plomb dans des moules issues du lac de Madine, une réserve d'eau potable pour la Ville de Metz, peut s'expliquer par le fait que ce site subit depuis plus d'un siècle les retombées de la pollution atmosphérique d'origine sidérurgique.
La concordance entre les teneurs en cuivre dans les dreissènes et les concentrations dans la colonne d'eau (Fig. 5) rend possible le calcul d'un flux de ce métal dans la retenue du Mirgenbach. En effet, lorsqu'on prend en compte les mesures mensuelles entre mai 1991 et février 1992 encadrant la période de prélèvement dans les autres compartiments, 


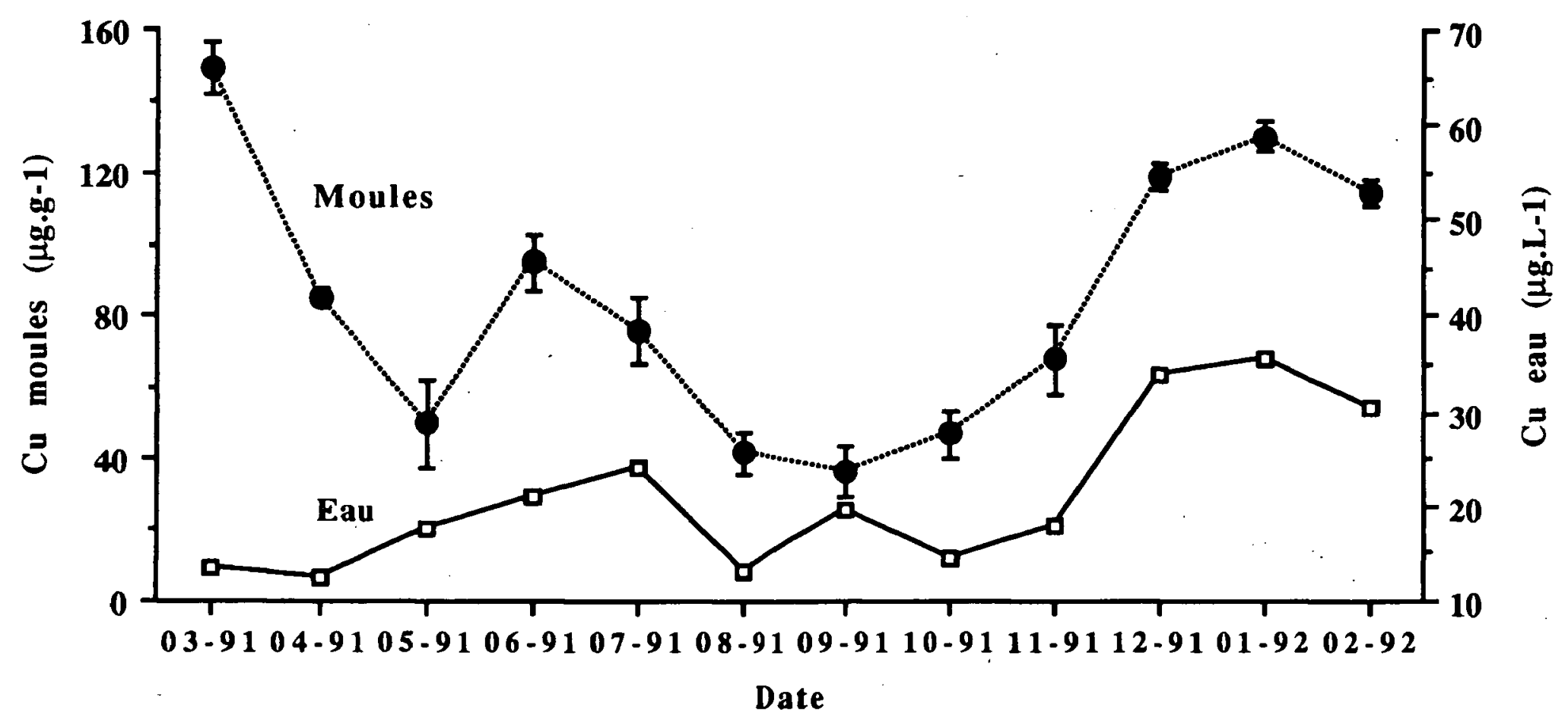

Fig. 5. Evolution mensuelle des concentrations en $\mathrm{Cu}$ dans l'eau $\left(\mu \mathrm{g} .1^{-1}\right)$ et dans la moule Dreissena polymorpha ( $\mu \mathrm{g} . \mathrm{g}^{-1}$ de poids sec) de la retenue du Mirgenbach. Les barres verticales représentent la variabilité entre deux sous-échantillons de 12 moules.

Fig. 5. Monthly changes of $\mathrm{Cu}$ concentrations in the water $\left(\mu \mathrm{g} . \mathrm{I}^{-1}\right)$ and in the mussel Dreissena polymorpha $\left(\mu \mathrm{g} . \mathrm{g}^{-1} \mathrm{dry}\right.$ weight) from the Mirgenbach reservoir. The vertical bars show the range between two sub-samples of 12 mussels each.

on obtient une droite de régression avec un coefficient de corrélation de 0.92 , significatif à $1 \%(n=$ 10). Cette relation souligne la représentativité des échantillons d'eau pendant cette période. La concentration moyenne dans l'eau entre juillet et décembre étant de $20.5 \pm 7.6 \mu \mathrm{g} .1-1$ et le temps moyen de séjour de l'eau étant d'environ 10 jours, le rejet de cuivre en sortie de la retenue dans la Moselle peut être estimé à $450 \pm 167 \mathrm{~kg}$ par mois.

\subsection{Poissons}

Le gardon accumule préférentiellement le cuivre et le zinc, alors que les teneurs en cadmium et en plomb sont plus élevées chez la perche (Fig. 6). Les concentrations observées dans le muscle sont en général très faibles; les différences statistiques observées entre les poissons des deux sites d'étude ne reflètent pas toujours la situation de pollution décrite par les autres supports. Ainsi, le cuivre dans le muscle de perche, mais aussi le plomb et le zinc dans celui de gardon sont significativement plus élevés dans les individus de l'étang de Lindre. Seul le cadmium est détecté dans le muscle des perches de la retenue à des concentrations plus élevées que dans celles de l'étang de Lindre. Par contre, les organes cibles des métaux, foie $(\mathrm{Cu})$ et rein $(\mathrm{Cd}, \mathrm{Pb}, \mathrm{Zn})$, fournissent des réponses exploitables avec des différences selon l'espèce. En ce qui concerne le foie, les concentrations dans les individus de la retenue sont significativement plus élevées chez le gardon pour le cuivre, chez la perche pour l'ensemble des 4 métaux. Dans le rein, les concentrations dans les individus de la retenue sont plus élevées chez le gardon pour le cadmium et chez la perche pour le cadmium et le plomb. L'unique cas inverse, c'est-à-dire avec une concentration plus élevée dans les poissons de l'étang que dans ceux de la retenue, est détecté pour le zinc dans le foie de gardon. Comme pour les autres supports, ces constats pourraient être affinés par comparaison avec des données de référence. Cependant, à cause de l'hétérogénéité des données bibliographiques, notamment en ce qui concerne l'expression des résultats (souvent donnés par rapport au poids frais) et le niveau de contamination des sites étudiés, il est difficile de proposer des concentrations de référence pour chacune des espèces et chacun des organes étudiés.

\section{Discussion}

L'objectif de la plupart des travaux sur la répartition et les transferts d'une contamination métallique dans un écosystème consiste à établir un constat d'une pollution ou à évaluer les risques sanitaires liés à un rejet industriel ou à un traitement chimique 

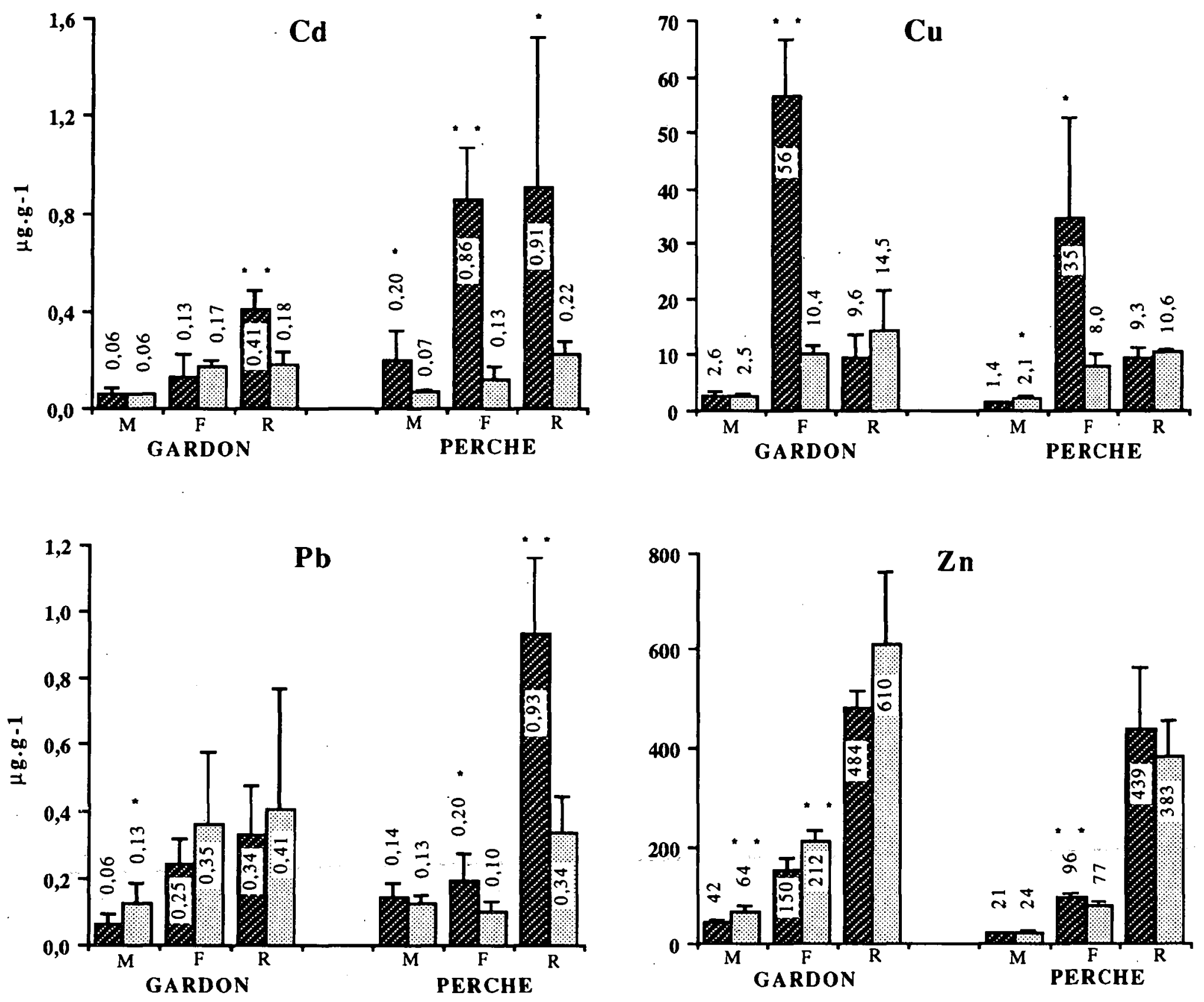

Fig. 6. Concentrations en $\mathrm{Cd}, \mathrm{Cu}, \mathrm{Pb}$ et $\mathrm{Zn}$ dans le muscle (M), le foie (F) et le rein (R) de gardon Rutilus rutilus et de perche Perca fluviatilis ( $\mu \mathrm{g} \cdot \mathrm{g}^{-1}$ de poids sec) de la retenue du Mirgenbach (barres foncées ; $n=5$ pour les deux espèces) et de l'étang de Lindre (barres claires ; $n=4) ;^{*}: p<0.05,{ }^{* *}: p<0.01$.

Concentrations of $\mathrm{Cd}, \mathrm{Cu}, \mathrm{Pb}$ and $\mathrm{Zn}$ in the muscle $(\mathrm{M})$, the liver $(\mathrm{F})$ and the kidney $(\mathrm{R})$ of the roach Rutilus rutilus and the perch Perca fluviatilis ( $\mu \mathrm{g} \cdot \mathrm{g}^{-1}$ dry weight) from the Mirgenbach reservoir (dark bars ; $n=5$ for both species) and from the Lindre pond (ligth bars $; n=4) ;^{*}: p<0.05,{ }^{* *}: p<0.01$.

(Nakanishi et al. 1989, Tanner \& Clayton 1990, Miller et al. 1992). Les commentaires sur l'intérêt comparé et la signification écotoxicologique des différents supports analytiques utilisés restent le plus souvent succincts. En outre, la comparaison avec les données disponibles est rendue difficile, du fait des particularités de la retenue du Mirgenbach, la plus singulière étant le taux de renouvellement moyen de l'eau, de l'ordre de 10 jours. De ce fait, ce réservoir possède un régime hydrologique intermédiaire entre celui d'un lac et celui d'une rivière.

Le cuivre, polluant métallique majeur de la retenue du Mirgenbach, est bien détecté dans les échantillons mensuels d'eau brute. La corrélation entre les concentrations instantanées de cuivre dans l'eau et celles des moules zébrées montre que la contamination a été chronique pendant la période de l'étude. Les différences des teneurs en cadmium, plomb et 
zinc entre la retenue du Mirgenbach et l'étang de Lindre ne peuvent être expliquées uniquement par un effet de concentration de l'eau pompée en Moselle, car elles dépassent le facteur moyen de 1.4 observé dans le cas des ions $\mathrm{Na}^{+}$et $\mathrm{Cl}^{-}$et de la conductivité. Il y a synergie de trois influences : un apport métallique de la Moselle, l'effet de l'évaporation et une libération additionnelle de métaux par corrosion du circuit de refroidissement de la centrale.

Dans l'eau, les différents métaux s'associent aux matières en suspension à des taux plus ou moins élevés. L'ordre d'affinité observé dans la retenue est le suivant : $\mathrm{Pb}>\mathrm{Zn} \geqslant \mathrm{Cu}>\mathrm{Cd}$. La fixation d'une fraction importante du plomb aux matières en suspension et, au contraire, la dominance de la forme libre du cadmium ont été observées dans d'autres milieux d'eau douce (Hart \& Davies 1981, Moore \& Ramamoorthy 1984, Pardo et al. 1989). En revanche, la spéciation du cuivre est beaucoup plus diversifiée et semble être fortement influencée par les caractéristiques physico-chimiques de l'eau (Bodo 1989). Dans la retenue du Mirgenbach, les fractions particulaires du cuivre et du zinc sont relativement faibles par rapport à celles observées dans d'autres milieux (Hart \& Davies 1981, Malle 1990). Ceci peut s'expliquer par la décantation rapide d'une partie des matières en suspension. Le comportement propre de chacun des métaux influence son devenir dans l'écosystème, notamment son transport vers les sédiments.

Le compartiment le plus pollué du réservoir est celui des sédiments. Pour chacun des quatre métaux, les concentrations observées dans la retenue du Mirgenbach dépassent nettement les niveaux de référence. L'étude du seston décanté montre que l'importation de micropolluants métalliques dans le réservoir est beaucoup plus importante que ne le suggéraient les analyses d'eau. En effet, les concentrations mesurées dans les échantillons d'eau représentent uniquement la fraction métallique qui est maintenue dans la phase circulante. Par conséquent, les matières en suspension, inertes ou biologiques, représentent, par leur importante capacité de piégeage de micropolluants, un maillon intermédiaire entre la phase circulante et la phase stationnaire, en l'occurence les sédiments. D'après Kaiser et al. (1989), les particules algales jouent un rôle déterminant dans l'élimination des métaux de la colonne d'eau par décantation. Dans la plupart des cas de pollution métallique, le sédiment représente le compartiment le plus fortement contaminé (Tanner \& Clayton 1990, Miller et al. 1992). Lorsqu'il y a remise en suspension de sédiment, ce compartiment est également une source de contamination de l'eau (Nakanishi et al. 1989, Tanner \& Clayton 1990, AlShukry et al. 1992).

Le cumul des dépôts de seston décanté permet le calcul d'un flux de métal représentatif d'une période donnée. Dans le cas du cuivre, une estimation de flux a également été possible pour la phase circulante du fait de la représentativité des échantillons d'eau, vérifiée par la corrélation avec le niveau de contamination des moules. Dans une zone de la retenue qui n'est pas influencée directement par l'injection des eaux échauffées et pour des échantillons d'eau prélevés en surface, ces deux flux peuvent être considérés comme complémentaires. En effet, on peut estimer que la séparation entre les solides susceptibles de décanter et ceux demeurant en suspension s'est achevée. Les flux de cuivre montrent que la majeure partie de ce métal, $938 \pm 474 \mathrm{~kg}$.mois ${ }^{-1}$ (68\% de la quantité totale), est retirée de la phase circulante contre $450 \pm 167 \mathrm{~kg} \cdot$ mois $^{-1}(32 \%)$ qui restent dans la colonne d'eau. Cette dernière valeur est en accord avec des résultats antérieurs évaluant à $2000 \mathrm{~kg} \cdot \mathrm{an}^{-1}$ la quantité de cuivre rejetée dans la Moselle pour deux réacteurs en fonctionnement (Mersch et al. 1992). Cette estimation ne tient compte que de la fraction métallique maintenue dans la colonne d'eau. Or, la charge totale de cuivre entrant dans la retenue du Mirgenbach correspond à la somme des deux fractions, évaluée ici à plus de $1300 \mathrm{~kg}$ par mois pour quatre réacteurs.

Les deux indicateurs biologiques, bryophytes et mollusques, fournissent des résultats qualitatifs très similaires sur la contamination de la colonne d'eau de la retenue. Ceci est remarquable pour deux organismes aussi différents. Le mécanisme d'accumulation des métaux par les mousses est principalement une adsorption (Caines et al. 1985, Breuer \& Melzer 1990). Il n'est pas propre aux mousses, mais existe pour d'autres surfaces biologiques, notamment les algues (Xue et al. 1988) et les filaments mycéliens (Siegel et al. 1990). L'adsorption permet une cinétique d'échange très rapide, ce qui a conduit à l'utilisation des mousses comme indicateurs de pollutions du type accidentel (Kelly et al. 1987, Mouvet et al. 1992). Un autre avantage des échanges de surface est la faible influence de la physiologie de 
l'organisme sur l'accumulation. En revanche, les mousses ne renseignent pas au sens strict sur la réelle biodisponibilité des métaux.

Le rôle bioindicateur des mollusques filtreurs a été abondamment mis en évidence (Fischer 1983, Léglize \& Crochard 1987, Abaychi \& Mustapha 1988). Ces organismes accumulent le métal à l'intérieur des cellules de différents organes cibles (Benyahia et al. 1988, Herwig et al. 1989). Un modèle d'homéostasie des métaux dans la glande digestive de la moule marine Mytilus edulis a été proposé par George \& Viarengo (1985). La capacité de régulation métabolique explique l'unique différence notable, observée pour le zinc, entre les moules et les mousses. En effet, l'écart de concentration entre le réservoir du Mirgenbach et le site témoin de la Meuse amont est plus prononcé pour les bryophytes que pour les mollusques. La capacité de régulation du zinc a été signalée pour des espèces de moules marines (Chan 1988, Martincic et al. 1992). La moule zébrée est capable de réguler efficacement ses niveaux intracellulaires de cuivre jusqu'à une concentration dans l'eau de $13 \mu \mathrm{g} . \mathrm{L}^{-1}$, au delà de ce seuil, le cuivre est accumulé (Kraak et al. 1992). En revanche, le cadmium est accumulé dès qu'il est présent à de faibles teneurs dans l'eau et le relargage est très lent (Bias \& Karbe 1985, Kraak et al. 1992). La régulation ou, au contraire, un processus cumulatif impliquent la prise en compte de l'impact biologique de chaque élément, puisque la concentration dans le mollusque ne reflète plus nécessairement celle dans l'eau. Dans le cas du cuivre, la corrélation entre les teneurs dans l'eau et celles dans les moules zébrées suggère qu'il existe un équilibre dynamique entre l'accumulation et l'élimination (recherche de l'homéostasie).

Dans le muscle de gardon et de perche, les teneurs métalliques ainsi que les différences entre la retenue du Mirgenbach et l'étang de Lindre restent très faibles. Ce tissu ne peut pas être considéré comme indicateur de la contamination du milieu. Contrairement au muscle, les organes de métabolisation et d'excrétion (foie et rein) présentent des propriétés accumulatrices de micropolluants (Sprenger et al. 1988, Miller et al. 1992). Dans le présent travail, seul le foie de perche fournit des indications contrastées entre la retenue et le site témoin. Par rapport à d'autres compartiments d'un écosystème, les niveaux métalliques restent globalement faibles dans les organescibles des poissons (Radwan et al. 1990, Miller et al. 1992, Baudin et al. 1991). Ceci s'explique par des capacités de régulation et d'adaptation des poissons à des situations de contamination (Hodson 1988, Sprenger et al. 1988, Carpenè et al. 1990).

\section{Conclusion}

L'analyse de six compartiments de la retenue du Mirgenbach, l'eau brute, les matières en suspension, le seston décanté, une mousse, une moule et des poissons, montre la pénétration des micropolluants métalliques à tous les niveaux, inertes et biologiques, de l'écosystème. Le contaminant majeur du site est le cuivre, associé au plomb et au zinc qui ont également été détectés à des teneurs supérieures aux niveaux de référence dans plusieurs compartiments. Le cadmium a essentiellement été trouvé dans les matières décantées.

La comparaison entre un site contaminé et un site dépourvu de pollution permet d'établir un constat fiable et, surtout, renseigne sur la dynamique et le devenir des contaminants dans les différents compartiments. Dans la retenue, une proportion importante des métaux entrants est piégée dans les sédiments. La répartition du métal entre la colonne d'eau et le seston décanté suggère que les analyses de la phase circulante et de la phase stationnaire sont complémentaires. Dans le cas du cuivre, les estimations indiquent que la relative dépollution de l'eau par sédimentation représente les deux tiers du flux de ce métal dans la retenue. Un tiers seulement, soit environ $450 \mathrm{~kg}$ par mois, reste dans la phase circulante. Il faut préciser que cette valeur moyenne se rapporte à la période d'étude entre juillet et décembre 1991, caractérisée par des concentrations de cuivre dans l'eau relativement faibles.

En ce qui concerne l'impact sur le milieu naturel, il faut s'intéresser principalement à la phase circulante qui est celle rejetée en rivière. Parmi les organismes testés, la mousse aquatique Fontinalis antipyretica et la moule zébrée Dreissena polymorpha ont confirmé leurs propriétés d'indicateurs biologiques de la contamination métallique circulante. Des indications homogènes obtenues avec deux organismes dont les mécanismes d'accumulation sont fondamentalement différents, peuvent être considérées comme particulièrement fiables. Les poissons, par contre, possèdent un système de régulation efficace qui maintient les concentrations des métaux étudiés à des niveaux faibles. Ceci implique qu'à l'exception des organes cibles, en particulier le foie de 
perche, le poisson apparaît comme un indicateur moins performant.

L'analyse comparée montre que chacun des compartiments étudiés possède des caractéristiques propres, avantages et inconvénients, pour évaluer la contamination métallique d'un écosystème. D'une part, l'interprétation des indications devient de plus en plus compliquée à mesure que le descripteur devient plus complexe. D'autre part, une donnée sur la contamination d'un organisme complexe possède un intérêt écotoxicologique plus élevé qu'une donnée concernant la concentration d'un polluant dans l'eau. Ceci pose le problème plus général du choix des compartiments à analyser. Ce choix dépend de plusieurs facteurs, comme le type de site étudié, l'origine et le type de la pollution, les moyens disponibles et le but recherché. L'objectif d'un travail peut être un constat d'une situation de contamination, le suivi de l'impact d'un rejet ou encore une surveillance sanitaire. Dans le cas de la retenue du Mirgenbach, les analyses du seston décanté (cumul du métal particulaire piégé) et des tissus de la moule zébrée (renseignant sur la contamination moyenne de la colonne d'eau) permettent un suivi efficace du niveau de pollution par les métaux.

\section{Remerciements}

Les auteurs remercient EDF, CPN de Cattenom. et le Domaine Piscicole de Lindre pour leur contribution à ce travail.

\section{Travaux cités}

Abaychi J.K. \& Mustafa Y.Z. 1988. - The asiatic clam, Corbicula fluminea : An indicator of trace metal pollution in the Shatt al-Arab River, Iraq. Environ. Pollut., 54 : 109-122.

Agence de l'Eau Rhin-Meuse. 1991. - Indicateurs de qualité des eaux superficielles: valeurs de référence pour divers métaux et micropolluants organiques. B.P. 19, 57161 Moulinslès-Metz Cedex, France : $178 \mathrm{p}$.

Al-Shukry R., Serpaud B., Matejka G. \& Caullet C. 1992. Spéciation des métaux lourds dans les sédiments d'un cours d'eau en aval d'un rejet industriel. Environ. Technol., 13 : 129-140.

Baudin J.P., Lambrechts A. \& Pally M. 1991. - Utilisation des mousses aquatiques comme bioindicateurs de contamination radioactive. Hydroécol. Appl., 3 : 209-240.

Batley G.E. 1987. - Heavy metal speciation in waters, sediments and biota from Lake Macquarie, New South Wales. Aust. J. Mar. Freshw. Res., 38 : 591-606.

Benyahia M., Crochard C. \& Pihan J.C. 1988. - Toxicité, bioaccumulation et relargage du plomb chez Dreissena polymorpha. Effet de l'EDTA et des phosphates. Haliotis, 18 : 239-249.
Bias R. \& Karbe L. 1985. - Bioaccumulation and partitioning of cadmium within the freshwater mussel Dreissena polymorpha Pallas. Int. Rev. gesamten Hydrobiol., 70 : 113-125.

Bodo B.A. 1989. - Heavy metals in water and suspended particulates from an urban basin impacting Lake Ontario. Sci Total Environ., 87/88 : 329-344.

Breuer K. \& Melzer A. 1990. - Heavy metal accumulation (lead and cadmium) and ion exchange in three species of Sphagnaceae I. Main principles of heavy metal accumulation in Sphagnaceae. Oecologia, $82:$ 461-467.

Caines L.A., Watt A.W. \& Wells D.E. 1985. - The uptake and release of some trace metals by aquatic bryophytes in acidified waters in Scotland. Environ. Pollut. Ser. B., $10: 1-18$.

Carpenè E., Cattani O., Serrazanetti G.P., Fedrizzi G. \& Cortesi P. 1990. - Zinc and copper in fish from natural waters and rearing ponds in nothern Italy. J. Fish Biol., 37 :293-299.

Chan H.M. 1988. - Accumulation and tolerance to cadmium, copper, lead and zinc by the green mussel Perna viridis. Mar. Ecol. Prog. Ser., 48 : 295-303.

Fischer H. 1983. - Shell weight as an independent variable in relation to cadmium content of molluscs. Mar. Ecol. Prog. Ser., 12 : 59-75.

Förstner U. \& Wittmann G.T.W. 1981. - Metal pollution in the aquatic environment. 2nd ed.; Springer Verlag, Berlin : $486 \mathrm{p}$.

George S.G. \& Viarengo A. 1985. - A model for heavy metal homeostasis and detoxication in mussel : 125-143, In Vernberg F.J., Thurberg F.P., Calabrese A., Vernberg W.B. (eds.). Marine pollution and physiology, Recent advances. Columbia, Univ. of South Carolina Press.

González H., Ramírez M. \& Ablanedo N. 1991. - Heavy metals in fish (Micropogonias furnieri) from Cienfuegos Bay, Cuba. Mar. Pollut. Bull., 22 : 469-471.

Guerrin F,, Burgat-Sacaze V. \& de Saqui-Sannes P. 1990. Levels of heavy metals and organochlorine pesticides of cyprinid fish reared four years in a wastewater treatment pond. Bull. Environ. Contam. Toxicol., 44 : 461-157.

Hart B.T. \& Davies S.H.R. 1981. - Trace metal speciation in the freshwater and estuarine regions of the Yarra River, Victoria. Estuar. Coast. Shelf Sci., 12 : 353-374.

Herwig H.J., Brands F., Kruitwagen E. \& Zandee D.I. 1989. Bioaccumulation and histochemical localization of cadmium in Dreissena polymorpha exposed to cadmium chloride. Aquat. Toxicol., $15: 269-286$.

Hodson P.V. 1988. - The effect of metal metabolism on uptake, disposition and toxicity in fish. Aquat. Toxicol., 11 : 3-18.

Kaiser M., Irmer U. \& Weiler K. 1989. - Monitoring of water quality : seasonal variations of heavy metals in sediment, suspended particulate matter and tubificids of the Elbe River. Environ. Technol. Lett., $10: 845-854$.

Kelly M.G., Girton C. \& Whitton B.A. 1987. - Use of mossbags for monitoring heavy metals in rivers. Water Res., 21 : 1429-1435.

Kraak M.H.S., Scholten M.C.T., Peeters W.H.M. \& de Kock W.C. 1991. - Biomonitoring of heavy metals in the Western European Rivers Rhine and Meuse using the freshwater mussel Dreissena polymorpha. Environ. Pollut., 74 : 101-114.

Kraak M.H.S., Lavy D., Peeters W.H.M. \& Davids C. 1992. Chronic ecotoxicity of copper and cadmium to the zebra mussel Dreissena polymorpha. Arch. Environ. Contam. Toxicol., 23 : 363-369. 
Léglize L. \& Crochard C. 1987. - Vérification expérimentale du choix de Dreissena polymorpha Pallas (lamellibranche) comme bioindicateur de contamination métallique. Nat. Can. (Rev. Ecol. Syst.), 114 : 315-323.

Malle K.G. 1990. - Metallgehalt und Schwebstoffgehalt im Rhein III. Z. Wasser-Abwasser-Forsch., 23 : 204-208.

Martincic D., Kwokal Z., Peharec Z., Margus D. \& Branica M. 1992. - Distribution of $\mathrm{Zn}, \mathrm{Pb}, \mathrm{Cd}$ and $\mathrm{Cu}$ between seawater and transplanted mussels (Mytilus galloprovincialis). Sci Total Environ., $119: 211-230$.

Mersch J., Jeanjean A., Spor H. \& Pihan J.C. 1992. - The freshwater mussel Dreissena polymorpha as a bioindicator for trace metals, organochlorines and radionuclides. In Neumann D. \& Jenner H.A. (eds), The zebra mussel Dreissena polymorpha. Fischer-Verlag, Stuttgart, Limnol. Aktuell, 4 : 227-244.

Miller P.A., Munkittrick K.R. \& Dixon D.G. 1992. Relationship between concentrations of copper and zinc in water, sediment, benthic invertebrates, and tissues of white sucker (Catostomus commersoni) at metal-contaminated sites. Can. J. Fish. Aquat. Sci., 49 : 978-984.

Moore J.W. \& Ramamoorthy S. 1984. - Heavy metals in natural waters. Applied monitoring and impact assessment. Springer-Verlag, New-York : $261 \mathrm{p}$.

Mouvet C. 1986. - Métaux lourds et mousses aquatiques : synthèse méthodologique. Rapport de contrat Université de Metz, Laboratoire d'Ecologie/Agence de l'Eau RhinMeuse/Agence de l'Eau Rhône-Méditerranée-Corse : 104 p.

Mouvet C., Morhain E., Sutter C. \& Couturieux N. 1992. Aquatic mosses for the detection and follow-up of accidental discharges in surface waters. Water Air Soil Pollut. 66 : 333-348.

Nakanishi H., Ukita M., Sekine M. \& Murakami S. 1989. Mercury pollution in Tokuyama Bay. Hydrobiologia, 176/177 : 197-211.
Pardo R., Barrado E., Arranz A., Pérez J.M. \& Vega M. 1989. - Levels and speciation of heavy metals in waters of Valladolid. Int. J. Environ. Anal. Chem., 37 : 117-123.

Pfeiffer W.C., de Lacerda L.D., Malm O., Souza C.M.M., da Silveira E.G. \& Bastos W.R. 1989. - Mercury concentrations in inland waters of gold-mining areas in Rondônia, Brazil. Sci. Total Environ., 87/88 : 233-240.

Radwan S., Kowalik W. \& Kornijow R. 1990. - Accumulation of heavy metals in a lake ecosystem. Sci. Total. Environ., $96: 121-129$.

Siegel S.M., Galun M. \& Siegel B.Z. 1990. - Filamentous fungi as metal biosorbents : A review. Water Air Soil Pollut., 53 : 335-344.

Sprenger M.D., McIntosh A.W. \& Hoenig S. 1988. - Concentrations of trace elements in yellow perch (Perca flavescens) from six acidic lakes. Water Air Soil Pollut., 37 : 375-388.

Swedish Environmental Protection Agency. 1991. - Quality criteria for lakes and watercourses. A system for classification of water chemistry and sediment and organism metal concentrations. 17185 Solna, Sweden : $32 \mathrm{p}$.

Tanner C.C. \& Clayton J.S. 1990. - Persistence of arsenic 24 years after sodium arsenite herbicide application to Lake Rotoroa, Hamilton, New Zealand. N.Z.J. Mar. Freshw. Res., 24 : 173-179.

Tessier A. \& Campbell P.G.C. 1987. - Partitioning of trace metals in sediments : relationships with bioavailability. Hydrobiologia, 149 : 43-52.

Villarreal-Treviño C.M., Obregón-Morales M.E., LozanoMorales J.F. \& Villegas-Navarro A. 1986. -Bioaccumulation of lead, copper, iron, and zinc by fish in a transect of the Santa Catarina River in Cadereyta Jiménez, Nuovo León, México. Bull. Environ. Contam. Toxicol., 37 : 395-401.

Wehr J.D., Empain A., Mouvet C., Say P.J. \& Whitton B.A. 1983. - Methods for processing aquatic-mosses used as monitors of heavy metals. Water Res., 17 : 985-992.

Xue H.B., Stumm W. \& Sigg L. 1988. - The binding of heavy metals to algal surfaces. Water Res., 22 : 917-926. 